(2) Open Access Full Text Article

REVIEW

\title{
Intense pulsed light for evaporative dry eye disease
}

This article was published in the following Dove Press journal:

Clinical Ophthalmology

20 June 2017

Number of times this article has been viewed

\author{
Steven J Dell \\ Dell Laser Consultants, Austin, TX, \\ USA
}

Correspondence: Steven J Dell

Dell Laser Consultants, 90I South Mopac Expressway, Building 4, Suite 350, Austin, TX 78746, USA

Tel + I 5 I2 3470255

Email steven@dellmd.com

\begin{abstract}
There is a clear association between dry eye disease (DED) and skin inflammatory diseases occurring in close proximity to the eyelids, such as facial skin rosacea. Intense pulsed light (IPL) is widely accepted as a treatment for skin rosacea. A number of recent studies demonstrated that, in patients suffering from meibomian gland dysfunction (MGD), IPL therapy also reduces signs and symptoms of DED. Despite these encouraging results, in the context of DED and MGD, the mechanisms of action of IPL are not well understood. The purpose of this review was to raise the potential mechanisms of action and to discuss their plausibility.
\end{abstract}

Keywords: intense pulsed light, dry eye disease, meibomian gland dysfunction, skin rosacea

\section{Introduction}

Dry eye disease (DED) is "a multifactorial disease of the tears and ocular surface..." that afflicts hundreds of millions around the world. ${ }^{1}$ In the US alone, 40 million people are estimated to suffer from, or to be predisposed to, this debilitating condition. ${ }^{2}$ DED is mostly age related, ${ }^{1}$ but can also be triggered by refractive ${ }^{3,4}$ or cataract surgery. ${ }^{5-7}$ In addition, preexisting DED significantly increases the risk of prolonged or severe post-op signs and symptoms of dry eye..$^{8,9}$ Refractive and cataract surgery patients have high visual expectations, and increasingly sophisticated intraocular lens and corneal ablation designs heighten the importance of good ocular surface health. Success of refractive and cataract surgeries is therefore, in many cases, fundamentally dependent on effectively addressing preexisting or iatrogenic DED. The most common form of DED is evaporative, which is mainly due to meibomian gland dysfunction (MGD). ${ }^{10}$ Current standard of care of MGD includes anti-inflammatory drugs, warm compresses, and meibomian gland expression. ${ }^{11-13}$

There is a clear association between MGD and skin inflammatory diseases occurring in close proximity to the eyelids. A common example is facial skin rosacea. One in ten people are affected by this skin condition, with $>80 \%$ of these patients having concomitant MGD. ${ }^{14}$ In $20 \%$ of the cases, ocular signs precede skin rosacea ${ }^{15}$ - possibly suggesting that skin rosacea could already exist in a subclinical form.

Intense pulsed light (IPL) is widely accepted as a treatment for skin rosacea. ${ }^{16}$ More than a decade ago, Toyos et al noticed that facial skin rosacea patients treated with IPL reported a significant improvement in their dry eye symptoms. ${ }^{17}$ Since then, a number of studies confirmed that IPL therapy reduces both signs and symptoms of dry eye. ${ }^{18-23}$ In these studies, IPL therapy comprised several sessions given several weeks apart. Each session consisted of IPL pulses applied from tragus to tragus, just below the lower eyelids and including the nose, as illustrated in Figure 1. 


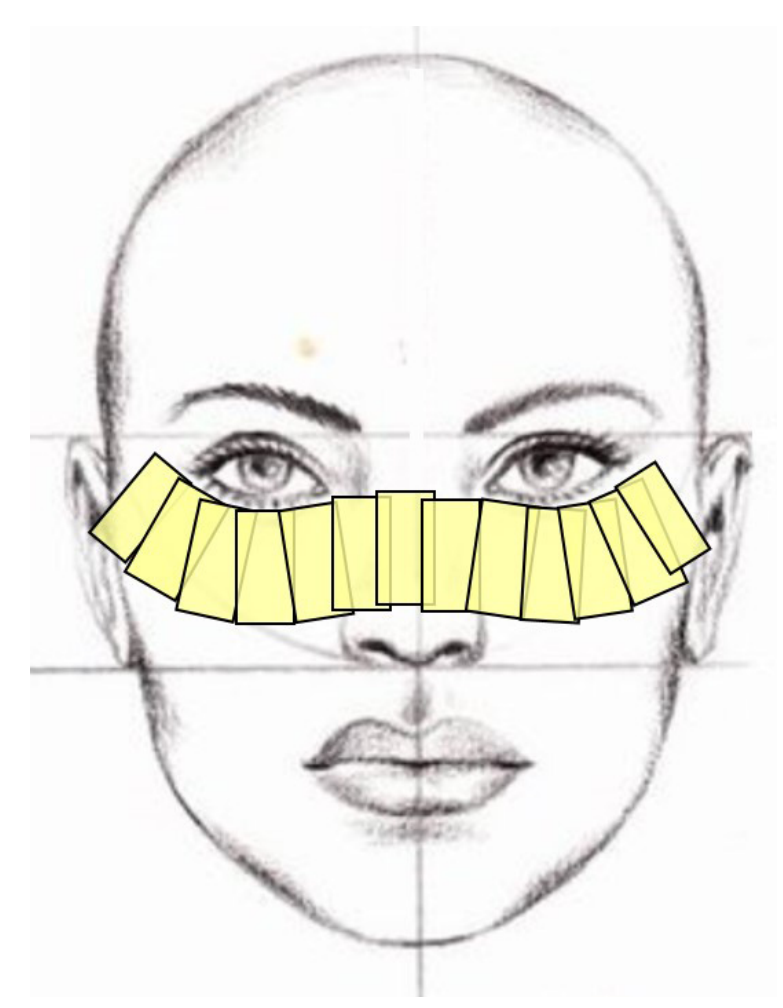

Figure I Treatment area in IPL therapy of MGD.

Note: Each yellow rectangle schematically represents the site of a single IPL pulse application.

Abbreviations: IPL, intense pulsed light; MGD, meibomian gland dysfunction.

Despite these encouraging results, the mechanism of action is not well understood. The purpose of this review is to raise the potential mechanisms of action and to discuss their plausibility.

\section{Thrombosis of abnormal blood vessels}

Facial skin rosacea is a chronic disorder presenting with vascular and inflammatory signs. The overwhelming majority of patients afflicted with this condition also suffer from MGD. ${ }^{14}$ Although the causal relationship is not entirely clear, it seems reasonable that MGD patients might benefit from treatment of their concomitant skin condition. One of the primary features of rosacea is skin erythema and telangiectasia. It has been proposed that these abnormal blood vessels release inflammatory mediators. ${ }^{18}$ Via the facial artery and orbital vasculature, these molecules could easily propagate to the eyelids, subsequently triggering the inflammation of meibomian glands and leading to their dysfunction and atrophy.

The beneficial effect of IPL on erythema and telangiectasia has been extensively studied and reported. ${ }^{16}$ Light energy absorbed by hemoglobin transforms to heat and causes the localized destruction of superficial blood vessels (thrombosis).
In the case of patients affected with MGD, destruction of abnormal erythematous blood vessels reduces a key reservoir of inflammatory mediators, thus removing a major source of inflammation from the eyelids and meibomian glands.

\section{Heating and liquefying the meibum}

Eyelid temperature significantly influences the physical properties of meibomian gland secretions, also known as meibum. ${ }^{24}$ At higher temperatures, meibum becomes less viscous, which more easily allows its normal distribution over the cornea. At room temperature, the temperature at the eyelids is $\sim 33^{\circ} \mathrm{C} .{ }^{25}$ In patients with MGD, lipid composition may be altered, reflecting changes in the configuration of hydrocarbon chain and lipid-lipid interaction strength. As a result, the phase-transition temperature (the temperature at which the meibomian lipids switch from an ordered and gellike phase to a disordered and fluid-like phase) may increase, compared to healthy subjects.

In a study that analyzed the physical properties of meibum, the phase-transition temperature was $\sim 28^{\circ} \mathrm{C}$ for meibum from healthy donors (below eyelid temperature), and just above $32^{\circ} \mathrm{C}$ for meibum from donors afflicted with MGD (above eyelid temperature). ${ }^{26}$ Because the phase-transition temperature of human meibum is near physiological body temperature, a small increase of $4^{\circ} \mathrm{C}$ is sufficient to change the meibum from gel like to fluid.

Indeed, warming the eyelids (with warm compresses or more sophisticated and automated devices) has some therapeutic value, as it facilitates meibomian gland expression. ${ }^{27}$ Craig et $\mathrm{a}^{19}$ noted that IPL application could induce an increase in skin temperature. However, these authors argued that any increase is modest and short lived: immediately after IPL application, the skin temperature increased by $<1{ }^{\circ} \mathrm{C} .{ }^{19}$ However, it should be noted that in their study, skin temperature was measured with infrared thermography a few seconds after treatment and only after removal of the conducting gel. During these few seconds, the skin could cool down considerably and lose heat. It is therefore difficult to infer from this measurement what the temperature of the eyelids would be during IPL treatment itself.

However, whether or not IPL energy is sufficient to warm the skin is less important than its thermal effect on blood vessels under the surface. The eyelids are extensively fed by capillaries and arterioles branching off the facial artery. A mathematical model demonstrates that in medium and large blood vessels ( $>150 \mu \mathrm{m}$ ), a single IPL pulse of $30 \mathrm{~ms}$ duration raises the temperature at the center of the vessel to $80^{\circ} \mathrm{C}-90^{\circ} \mathrm{C}$, above the temperature required to cause coagulation and 
thrombosis as discussed above. ${ }^{28}$ In contrast, in small $(60 \mu \mathrm{m})$ blood vessels, the temperature may reach only $45^{\circ} \mathrm{C}-70^{\circ} \mathrm{C}$, depending on fluence. ${ }^{28}$ This temperature elevation is insufficient to cause the destruction of blood vessels, but it is probably enough to raise the temperature of eyelid skin (and meibomian glands) by a few degrees, possibly above the phase-transition temperature. Even if brief, this thermal response could be enough to unclog the meibomian glands and restore their ability to excrete meibum during blinking.

\section{Reducing the epithelial turnover and decreasing the risk for gland obstruction}

As often occurs in skin diseases, cutaneous rosacea is accompanied by a dramatic increase in epithelial skin turnover. In a mechanism similar to dandruff production, large amounts of dead epithelial skin cells detach from the epidermal surface and create debris. Since the ducts of meibomian glands are paved with the same type of epithelial cells, accumulation of debris on the lid margin is likely to occur. This, in combination with poor lid hygiene, could potentially clog the orifices of meibomian glands. ${ }^{29}$ IPL treatment of rosacea could, thus, decrease the epithelial turnover and reduce the risk factor for obstruction.

\section{Photomodulation}

Photomodulation is a process by which light in the visible and infrared portions of the electromagnetic spectrum induces intracellular changes at the gene and protein levels. The biological basis of this process is not well understood. According to the Karu model, red $(\sim 630 \mathrm{~nm})$ photons are absorbed in cytochrome $\mathrm{C}$ oxidase (Cox), a key enzyme in the electron transport chain embedded within the membrane of mitochondria. Photoexcitation of Cox prompts a photochemical cascade, inducing changes in the redox properties of components along this mitochondrial respiratory chain, leading to quickened electron transfer and, hence, to an increase in ATP production. ${ }^{30,31}$ The cytoplasmic rise of ATP activates various intracellular/extracellular exchange mechanisms (pumps and transporters), resulting in an increase in intracellular free calcium concentration.

Smith proposes a complementary model, by which the absorption of infrared photons $(\sim 810 \mathrm{~nm})$ induces molecular rotations and vibrations of various molecules. ${ }^{32}$ When such physical forces are exerted on calcium channels, the permeability of these channels is altered such that the influx of calcium ions increases. Here as well, the end result is an abrupt surge in intracellular calcium concentration.
This calcium signal activates cellular responses in a variety of ways. In the case of fibroblasts, cell proliferation is enhanced and collagen synthesis is increased ${ }^{33}$ skin-homing T cells are recruited; ${ }^{34}$ local blood flow is increased; macrophages cells are activated; ${ }^{35}$ epidermal keratinocytes increase the secretion of proinflammatory or anti-inflammatory cytokines and chemokines, depending on the context.

\section{Activating fibroblasts and enhancing collagen synthesis}

The extracellular matrix comprises three types of fibers: collagen, reticular, and elastin. ${ }^{36}$ With age, all the three types of fibers relax to some extent, thus compromising the natural rigidity and elasticity of tissues. At the eyelid skin level, this process can lead to poor apposition of the lid margins and incomplete blinks, resulting in reduced meibum pumping out of the meibomian glands. This can lead in turn to increased tear evaporation.

Fibroblast cells are responsible for the production of collagen fibers in wound healing and tissue repair. As mentioned earlier, photomodulation can prompt the proliferation of fibroblasts and upregulate the synthesis of collagen fibers. ${ }^{33}$ An in vitro study showed that a pulsed $660 \mathrm{~nm}$ (LED) light enhanced collagen production in a tissue-engineered reconstructed skin model. ${ }^{37}$ In another in vitro study, irradiation of skin fibroblasts with IPL (800-1,200 nm) increased the proliferation rate of fibroblasts and increased the expression of collagen genes. ${ }^{38}$ These results are also supported by clinical studies. ${ }^{39}$

\section{Eradicating Demodex}

One of the potential mediators of blepharitis and MGD are Demodex folliculum mites, a type of ectoparasite that normally burrows deep into sebaceous and meibomian glands to feed on their sebum/meibum secretions. ${ }^{40}$ In healthy skin, the degree of infestation with Demodex mites is controlled. Demodex mites are normally colonized with Bacillus olerinus. ${ }^{41,42}$ Rosacea patients present with increased Demodex population on the face, high serum reactivity to B. olerinus proteins, and reduced levels of sebum. ${ }^{43}$

The causal relationship between rosacea and Demodex is not clear. Some researchers argue that rosacea is fundamentally an infectious disease resulting from Demodex thriving on skin damaged by a combination of age, adverse weathering, and changes in sebum composition. ${ }^{44}$ Others claim that erythema and superficial telangiectasia (which are characteristics of rosacea) induce edema of the dermis, which in turn increases skin colonization of Demodex. ${ }^{45}$ 
A direct consequence of Demodex proliferation is the dramatic increase in bacterial load on the eyelids, ${ }^{46}$ particularly B. olerinus. The excessive presence of $B$. olerinus near the eyelids triggers a cascade of events that may degenerate into chronic inflammation of the ocular surface. First, the immune system responds by orchestrating an army of proinflammatory agents, including antimicrobial peptides, toll-like receptors, cytokines, chemokines, and matrix metalloproteinases (MMPs). ${ }^{47,48}$ In small quantities, these agents may perform well. But an acute inflammatory response may turn into a chronic, self-perpetuating condition. Second, B. olerinus releases toxic substances, including lipases which enzymatically alter lipid composition. A change in the ratio of saturated to unsaturated fats of the meibum could raise its melting point, increase its viscosity, and impede its secretion. In addition, one by-product of lipase activity on sebum/ meibum is oleic acid, which could play a role in the keratinization of the lid margin, and plugging of the meibomian gland orifices. ${ }^{13}$ All of these events could aggravate and perpetuate inflammation inside the meibomian glands.

The pigmented exoskeleton of Demodex contains chromophore that absorbs IPL energy. Histologic analysis demonstrated that IPL treatment induces coagulation and necrosis of Demodex. ${ }^{49,50}$ By eradication of Demodex, IPL could decrease the microbial load on eyelids and potentially break the vicious cycle of inflammation.

\section{Modulating the secretion of pro- and anti-inflammatory molecules}

Inflammation has a pivotal role in the development and propagation of evaporative DED in early as well as advanced phases of the disease.${ }^{51}$ Factors that adversely affect tear film stability and osmolarity can induce ocular damage and initiate an inflammatory cascade that generates a powerful immunological response which, in turn, may cause further damage at the ocular surface, creating a self-perpetuating inflammatory cycle. Clinical studies consistently report elevated levels of inflammatory molecules in the tears and ocular surface of patients with DED. ${ }^{52}$ The levels of these cytokines/ chemokines are often correlated with pain, tear instability, tear production, and/or ocular surface integrity. ${ }^{51}$

IPL has the potential to interfere with this inflammatory cycle, by upregulation of anti-inflammatory cytokines, or downregulation of proinflammatory cytokines, or both. A few examples are noteworthy:

1. In cultured keratinocytes, IPL treatment led to a fivefold increase in the levels of interleukin-10 (IL-10), an antiinflammatory protein that inhibits cytokine production in
T cells. ${ }^{53}$ In fibroblasts, IPL has a bidirectional effect on the secretion of transforming growth factor- $\beta 1$ (TGF- $\beta 1$ ): inhibition at low fluences, but enhancement at high fluences. ${ }^{54}$ TFG- $\beta$ is an interesting example, because it has both pro- and anti-inflammatory effects, depending on the context and the cellular environment. As an antiinflammatory agent, TGF- $\beta$ modulates the proliferation of $\mathrm{T}$ cells after encountering ocular surface epithelium, prevents their migration to the conjunctiva, ${ }^{55}$ and suppresses natural killer (NK) cells.

2. A third example is the proinflammatory cytokine IL-6, which is downregulated subsequent to LED phototherapy. ${ }^{56}$

3. Yet another example is the effect of IPL on the skin of acne patients: IPL significantly reduces inflammatory lesions, presumably by downregulation of tumor necrosis factor- $\alpha$ (TNF- $\alpha$ ) (one of the cytokines which make up the acute phase of inflammation). ${ }^{57}$

The inflammatory cascade in dry eye is extremely complex and incompletely understood. However, it is plausible that at least part of the beneficial effect of IPL on DED patients occurs by interfering with the positive feedback loop underlying the inflammatory cycle of this pathology.

\section{Suppressing MMPs}

Another type of proteins involved in the pathogenesis of dry eye are MMPs. These enzymes participate in extracellular matrix remodeling and are both directly and indirectly affected by IPL. For example, in skin fibroblasts, IPL treatment decreases the concentration of MMPs, by downregulation at the mRNA level. ${ }^{58}$ In corneal epithelia cells, TNF- $\alpha$ and IL-1 upregulate several types of MMPs. ${ }^{59}$ Recall that TNF- $\alpha$ is downregulated by IPL. ${ }^{57}$ Therefore, IPL indirectly diminishes the levels of these MMPs. It is interesting to note that corticosteroids relieve dry eye symptoms by similar pathways: they interfere with the inflammatory cycle by lowering the cellular levels of cytokines, chemokines, and MMPs. ${ }^{60-62}$

\section{Reactive oxidative species (ROS)}

In rosacea, inflammation is associated with the generation of ROS released by neutrophils and other inflammatory cells. ${ }^{63}$ ROS are highly reactive molecules containing oxygen, also widely referred to as free radicals. Examples of ROS include superoxide anions $\left(\mathrm{O}_{2}^{-}\right)$and hydroxyl radicals $(\mathrm{OH}-)$. Abnormally high levels of ROS may result in oxidative stress, as was identified in the tear film of dry eye patients. ${ }^{64}$

There are conflicting reports regarding the effect of visible light irradiation on the levels of ROS. For example, absorption of visible light in mitochondrial and cell membrane 


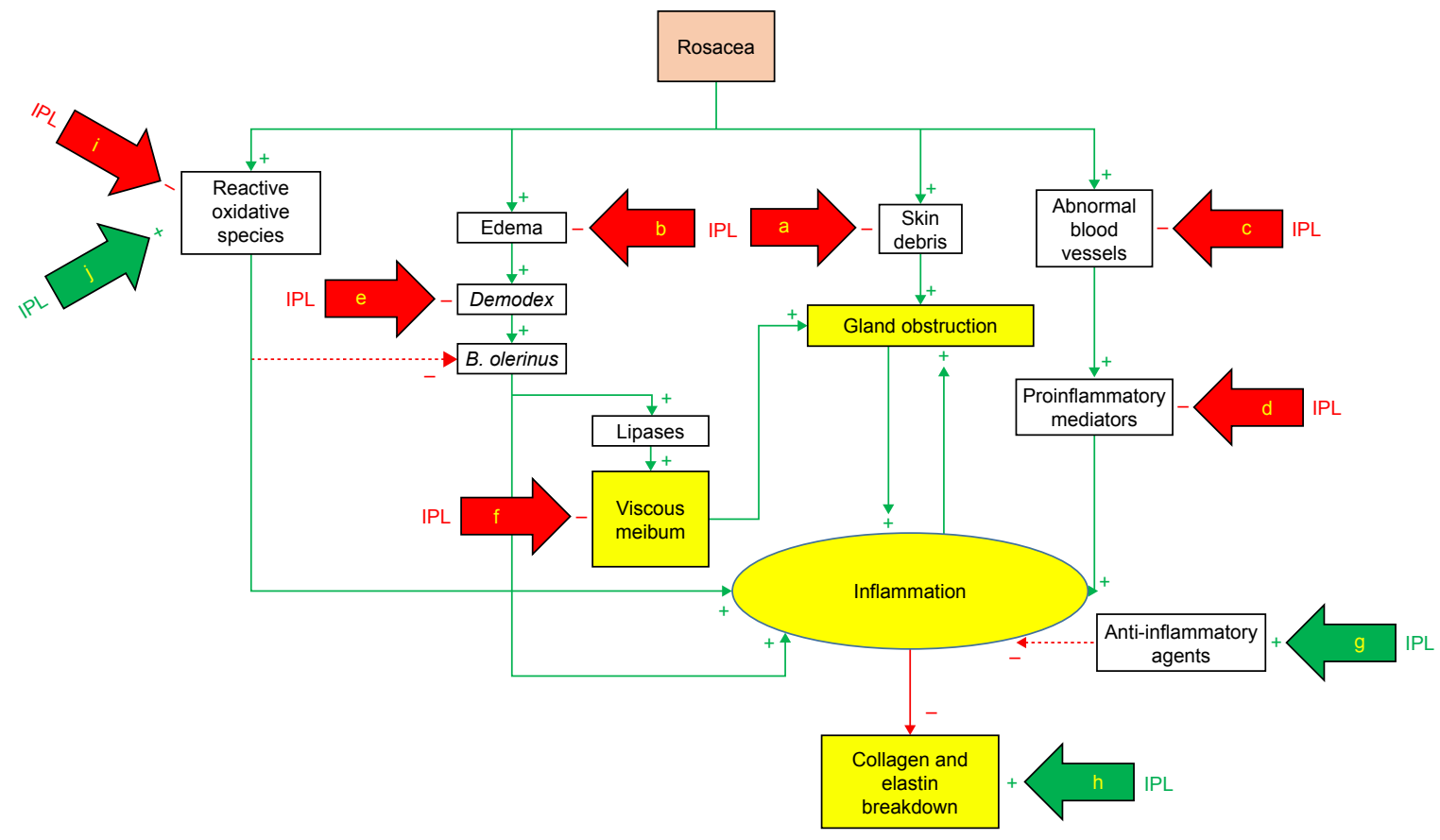

Figure 2 Mechanisms of action of IPL (simplified model).

Notes: Green arrows (+) represent effects that increase the level of the target; red arrows (-) represent effects that decrease the level of the target.

Abbreviations: IPL, intense pulsed light; a, skin rejuvenation; b, rosacea treatment; c, thrombosis; d, down-regulation; e, coagulation; f, warming and liquefying; g, up-regulation; h, fibroblasts activation; i, attenuation; j, production.

cytochromes generate ROS and thus could induce oxidative stress ${ }^{65}$ One report shows that application of light results in reduced levels of ROS. ${ }^{66}$ Several researchers have proposed that the effect of light on ROS levels follows a biphasic dose response, also known as the Arndt-Schultz curve. ${ }^{67,68}$

Separately, either one of these contradictory effects could have a beneficial effect on dry eye patients. Following lowlevel light irradiation, an increase in ROS is described by the ascending part of the Arndt-Schultz curve. In this situation, light irradiation would result in excessive production of ROS and antimicrobial activity, thus reducing the bacterial load on eyelids. At higher doses, the descending part of the Arndt-Schultz curve could describe the antioxidant roles of light irradiation. In this part of the dose-response curve, light irradiation would result in the attenuation of ROS levels, thus diminishing oxidative stress and inflammation.

\section{Conclusion}

Dry eye is a multifactorial disease. Potential mechanisms whereby IPL could achieve clinical improvement include thrombosis of abnormal blood vessels below the skin surrounding the eyes, heating the meibomian glands and liquefying the meibum, activation of fibroblasts and enhancing the synthesis of new collagen fibers, eradication of Demodex and decreasing the bacterial load on the eyelids, interference with the inflammatory cycle by regulation of anti-inflammatory agents and MMPs, reducing the turnover of skin epithelial cells and decreasing the risk of physical obstruction of the meibomian glands, and changes in the levels of ROS (Figure 2). While any one of these mechanisms of action has the potential to explain the effect of IPL on DED, it is also possible that multiple mechanisms of action are at play. As IPL becomes more commonly used in the treatment of DED, the specific contribution of each of these modes of action will be further elucidated.

\section{Disclosure}

SJD is a consultant for Lumenis Ltd. The author reports no other conflicts of interest in this work.

\section{References}

1. DEWS. The definition and classification of dry eye disease: report of the Definition and Classification Subcommittee of the International Dry Eye Workshop. Ocul Surf. 2007;5(2):75-92.

2. Ding J, Sullivan D. Aging and dry eye disease. Exp Gerontol. 2012;47(7):483-490.

3. Levitt A, Galor A, Weiss J, et al. Chronic dry eye symptoms after LASIK: parallels and lessons to be learned from other persistent post-operative pain disorders. Mol Pain. 2015;11:21.

4. Shoja M, Besharati M. Dry eye after LASIK for myopia: incidence and risk factors. Eur J Ophthalmol. 2007;17(1):1-6.

5. Li X, Hu L, Hu J, Wang W. Investigation of dry eye disease and analysis of the pathogenic factors in patients after cataract surgery. Cornea. 2007;26(9 Suppl 1):S16-S20.

6. Ang R, Dartt D, Tsubota K. Dry eye after refractive surgery. Curr Opin Ophthalmol. 2001;12(4):318-322. 
7. Roberts C, Elie E. Dry eye symptoms following cataract surgery. Insight. 2007;32(1):14-21.

8. Shtein R. Post-LASIK dry eye. Expert Rev Ophthalmol. 2011;6(5): $575-582$.

9. Cetinkaya S, Mestan E, Acir N, Cetinkaya Y, Dadaci Z, Yener H. The course of dry eye after phacoemulsification surgery. BMC Ophthalmol. 2015;15(68):1-5.

10. Lemp M, Crews L, Bron A, Foulks G, Sullivan B. Distribution of aqueous-deficient and evaporative dry eye in a clinic-based patient cohort. Cornea. 2008;27:1142-1147.

11. Thode A, Latkany R. Current and emerging therapeutic strategies for the treatment of meibomian gland dysfunction (MGD). Drugs. 2015; 75(11):1177-1185.

12. Ezuddin N, Alawa K, Galor A. Therapeutic strategies to treat dry eye in an aging population. Drugs Aging. 2015;32(7):505-513.

13. Geerling G, Tauber J, Baudouin C, et al. The international workshop on meibomian gland dysfunction: report of the subcommittee on management and treatment of meibomian gland dysfunction. Invest Ophthalmol Vis Sci. 2011;52(4):2050-2064.

14. Viso E, Rodríguez-Ares MD, Oubiña B, Gude F. Prevalence of asymptomatic and symptomatic meibomian gland dysfunction in the general population of Spain. IOVS. 2012;53(6):2601-2606.

15. Ghanem V, Mehra N, Wong S, Mannis M. The prevalence of ocular signs in acne rosacea: comparing patients from ophthalmology and dermatology clinics. Cornea. 2003;22(3):230-233.

16. Papageorgiou P, Clayton W, Norwood S, Chopra S, Rustin M. Treatment of rosacea with intense pulsed light: significant improvement and long-lasting results. Br J Dermatol. 2008;159(3):628-632.

17. Toyos R, Buffa C, Youngerman S. Case report: dry-eye symptoms improve with intense pulsed light treatment. Available from: www.eyeworld.org/article.php?sid=2698. EyeWorld (ASCRS). September 2005. Accessed February 15, 2015.

18. Toyos R, McGill W, Briscoe D. Intense pulsed light treatment for dry eye disease due to meibomian gland dysfunction: a 3-year retrospective study. Photomed Laser Surg. 2015;33(1):41-46.

19. Craig J, Chen Y, Turnbull P. Prospective trial of intense pulsed Light for the treatment of meibomian gland dysfunction. Invest Ophthalmol Vis Sci. 2015;56(3):1965-1970.

20. Vegunta S, Patel D, Shen J. Combination therapy of intense pulsed light therapy and meibomian gland expression (IPL/MGX) can improve dry eye symptoms and meibomian gland function in patients with refractory dry eye: a retrospective analysis. Cornea. 2016;35(3): 318-322.

21. Vora G, Gupta P. Intense pulsed light therapy for the treatment of evaporative dry eye disease. Curr Opin Ophthalmol. 2015;26:314-318.

22. Jiang X, Lv H, Song H, et al. Evaluation of the Safety and Effectiveness of Intense Pulsed Light in the Treatment of Meibomian Gland Dysfunction. J Ophthalmol. 2016;2016:1910694

23. Dell S, Gaster R, Barbarino S, Cunningham D. Prospective evaluation of intense pulsed light and meibomian gland expression efficacy on relieving signs and symptoms of dry eye disease due to meibomian gland dysfunction. Clin Ophthalmol. 2017;11:817-827.

24. Nagymihályi A, Dikstein S, Tiffany J. The influence of eyelid temperature on the delivery of meibomian oil. Exp Eye Res. 2004;78(3): 367-370.

25. Butovich I, Millar T, Ham B. Understanding and analyzing meibomian lipids-a review. Curr Eye Res. 2008;33(5):405-420.

26. Borchman D, Foulks G, Yappert M, et al. Human meibum lipid conformation and thermodynamic changes with meibomian-gland dysfunction. IOVS. 2011;52(6):3805-3817.

27. Finis D, Hayajneh J, König C, Borrelli M, Schrader S, Geerling G. Evaluation of an automated thermodynamic treatment (LipiFlow ${ }^{\mathbb{B}}$ ) system for meibomian gland dysfunction: a prospective, randomized, observer-masked trial. Ocul Surf. 2014;12:146-154.

28. Bäumler W, Vural E, Landthaler M, Muzzi F, Shafirstein G. The effects of intense pulsed light (IPL) on blood vessels investigated by mathematical modeling. Lasers Surg Med. 2007;39(2):132-139.
29. Henriquez A, Korb D. Meibomian glands and contact lens wear. $B r J$ Ophthalmol. 1981;65(2):108-111.

30. Karu T. Primary and secondary mechanisms of action of visible to nearIR radiation on cells. J Photochem Photobiol B. 1999;49(1):1-17.

31. Farivar S, Malekshahabi T, Shiari R. Biological effects of low level laser therapy. J Lasers Med Sci. 2014;5(2):58-62.

32. Smith $\mathrm{K}$. The photobiological basis of low level laser radiation therapy. Laser Ther. 1991;3:19-24.

33. Takezaki S, Omi T, Sato S, Kawana S. Ultrastructural observations of human skin following irradiation with visible red light-emitting diodes (LEDs): a preliminary in vivo report. Laser Ther. 2005;14(4): $153-160$.

34. Takezaki S, Omi T, Sato S, Kawana S. Light-emitting diode phototherapy at $630+/-3 \mathrm{~nm}$ increases local levels of skin-homing T-cells in human subjects. J Nippon Med Sch. 2006;73(2):75-81.

35. Young S, Bolton P, Dyson M, Harvey W, Diamantopoulos C. Macrophage responsiveness to light therapy. Lasers Surg Med. 1989;9(5): 497-505.

36. Ushiki T. Collagen fibers, reticular fibers and elastic fibers. A comprehensive understanding from a morphological viewpoint. Arch Histol Cytol. 2002;65(2):109-126.

37. Barolet D, Roberge C, Auger F, Boucher A, Germain L. Regulation of skin collagen metabolism in vitro using a pulsed $660 \mathrm{~nm}$ LED light source: clinical correlation with a single-blinded study. J Invest Dermatol. 2009;129(12):2751-2759.

38. Cuerda-Galindo E, Díaz-Gil G, Palomar-Gallego M, LinaresGarcíaValdecasas R. Increased fibroblast proliferation and activity after applying intense pulsed light 800-1200 nm. Ann Anat. 2015;198:66-72.

39. Goldberg D. Current trends in intense pulsed light. $J$ Clin Aesthet Dermatol. 2012;5(6):45-53.

40. Liu J, Sheha H, Tseng S. Pathogenic role of Demodex mites in blepharitis. Curr Opin Allergy Clin Immunol. 2010;10(5):505-510.

41. Szkaradkiewicz A, Chudzicka-Strugała I, Karpiński T, et al. Bacillus oleronius and Demodex mite infestation in patients with chronic blepharitis. Clin Microbiol Infect. 2012;18(10):1020-1025.

42. Li J, O'Reilly N, Sheha H, et al. Correlation between ocular Demodex infestation and serum immunoreactivity to Bacillus proteins in patients with facial rosacea. Ophthalmology. 2010;117(5):870-877.

43. Jarmuda $\mathrm{S}, \mathrm{McMahon} \mathrm{F}, \mathrm{Zaba} \mathrm{R}$, et al. Correlation between serum reactivity to Demodex-associated Bacillus oleronius proteins, and altered sebum levels and Demodex populations in erythematotelangiectatic rosacea patients. J Med Microbiol. 2014;63(Pt 2):258-262.

44. Jarmuda S, O'Reilly N, Zaba R, Jakubowicz O, Szkaradkiewicz A, Kavanagh K. Potential role of Demodex mites and bacteria in the induction of rosacea. J Med Microbiol. 2012;61(11):1504-1510.

45. Cribier B. Pathophysiology of rosacea: redness, telangiectasia, and rosacea. Ann Dermatol Venereol. 2011;138(Suppl 3):184-191.

46. O'Reilly N, Menezes N, Kavanagh K. Positive correlation between serum immunoreactivity to Demodex-associated Bacillus proteins and erythematotelangiectatic rosacea. Br J Dermatol. 2012;167(5): 1032-1036.

47. Margalit A, Kowalczyk M, Żaba R, Kavanagh K. The role of altered cutaneous immune responses in the induction and persistence of rosacea. J Dermatol Sci. 2016;82(1):3-8.

48. Lacey N, Delaney S, Kavanagh K, Powell F. Mite-related bacterial antigens stimulate inflammatory cells in rosacea. Br J Dermatol. 2007; 157(3):474-481.

49. Prieto V, Sadick N, Lloreta J, Nicholson J, Shea C. Effects of intense pulsed light on sun-damaged human skin, routine, and ultrastructural analysis. Lasers Surg Med. 2002;30(2):82-85.

50. Kirn T. Intense pulsed light eradicates Demodex mites. Skin Allergy News. 2002;33(1):37.

51. Enríquez-de-Salamanca A, Castellanos E, Stern M, et al. Tear cytokine and chemokine analysis and clinical correlations in evaporative-type dry eye disease. Mol Vis. 2010;16:862-873.

52. Stevenson W, Chauhan S, Dana R. Dry eye disease: an immune-mediated ocular surface disorder. Arch Ophthalmol. 2012;130(1):90-100. 
53. Byun J, Choi H, Myung K, Choi Y. Expression of IL-10, TGF- $\beta 1$ and TNF- $\alpha$ in cultured keratinocytes (HaCaT Cells) after IPL treatment or ALA-IPL photodynamic treatment. Ann Dermatol. 2009;21(1): $12-17$.

54. Huang J, Luo X, Lu J, et al. IPL irradiation rejuvenates skin collagen via the bidirectional regulation of MMP- 1 and TGF- $\beta 1$ mediated by MAPKs in fibroblasts. Lasers Med Sci. 2011;26(3):381-387.

55. De Paiva C, Volpe E, Gandhi N, et al. Disruption of TGF- $\beta$ signaling improves ocular surface epithelial disease in experimental autoimmune keratoconjunctivitis sicca. Plos One. 2011;6(12):e29017.

56. Lee S, Park K, Choi J, et al. A prospective, randomized, placebocontrolled, double-blinded, and split-face clinical study on LED phototherapy for skin rejuvenation: clinical, profilometric, histologic, ultrastructural, and biochemical evaluations and comparison of three different treatment settings. J Photochem Photobiol B. 2007;88(1): 51-67.

57. Taylor M, Porter R, Gonzalez M. Intense pulsed light may improve inflammatory acne through TNF- $\alpha$ down-regulation. J Cosmet Laser Ther. 2014;16(2):96-103.

58. Wong WR, Shyu WL, Tsai JW, Hsu KH, Lee HY, Pang JH. Intense pulsed light modulates the expressions of MMP-2, MMP-14 and TIMP-2 in skin dermal fibroblasts cultured within contracted collagen lattices. J Dermatol Sci. 2008;51(1):70-73.

59. Li D, Shang T, Kim H, Solomon A, Lokeshwar B, Pflugfelder S. Regulated expression of collagenases MMP-1, -8 , and -13 and stromelysins MMP-3, -10, and -11 by human corneal epithelial cells. Invest Ophthalmol Vis Sci. 2003;44:2928-2935.
60. Aragona P, Aguennouz M, Rania L, et al. Matrix metalloproteinase 9 and transglutaminase 2 expression at the ocular surface in patients with different forms of dry eye disease. Ophthalmology. 2015;122(1):62-71.

61. Byun Y, Kim T, Kwon S, et al. Efficacy of combined $0.05 \%$ cyclosporine and $1 \%$ methylprednisolone treatment for chronic dry eye. Cornea. 2012;31(5):509-513.

62. De Paiva C, Corrales R, Villarreal A, et al. Corticosteroid and doxycycline suppress MMP-9 and inflammatory cytokine expression, MAPK activation in the corneal epithelium in experimental dry eye. Exp Eye Res. 2006;83(3):526-535.

63. Jones D. Reactive oxygen species and rosacea. Cutis. 2009;74(Suppl 3): 17-20,32-34.

64. Augustin A, Spitznas M, Kaviani N, et al. Oxidative reactions in the tear fluid of patients suffering from dry eyes. Graefes Arch Clin Exp Ophthalmol. 1995;233(11):694-698.

65. Lubart R, Eichler M, Lavi R, Friedman H, Shainberg A. Low-energy laser irradiation promotes cellular redox activity. Photomed Laser Surg. 2005;23(1):3-9.

66. Lan C, Ho P, Wu C, Yang R, Yu H. LED $590 \mathrm{~nm}$ photomodulation reduces UVA-induced metalloproteinase-1 expression via upregulation of antioxidant enzyme catalase. J Dermatol Sci. 2015;78(2):125-132.

67. Lubart R, Lavi R, Friedmann H, Rochkind S. Photochemistry and photobiology of light absorption by living cells. Photomed Laser Surg. 2006;24(2):179-185.

68. Huang YY, Chen AH, Carroll J, MR H. Biphasic dose response in low level light therapy. Dose Response. 2009;7(4):358-383.
Clinical Ophthalmology

\section{Publish your work in this journal}

Clinical Ophthalmology is an international, peer-reviewed journa covering all subspecialties within ophthalmology. Key topics include: Optometry; Visual science; Pharmacology and drug therapy in eye diseases; Basic Sciences; Primary and Secondary eye care; Patien Safety and Quality of Care Improvements. This journal is indexed on

Submit your manuscript here: http://www.dovepress.com/clinical-ophthalmology-journal

\section{Dovepress}

PubMed Central and CAS, and is the official journal of The Society of Clinical Ophthalmology (SCO). The manuscript management system is completely online and includes a very quick and fair peer-review system, which is all easy to use. Visit http://www.dovepress.com/ testimonials.php to read real quotes from published authors. 